\title{
FABRICATION OF NEURAL MICROELECTRODE ARRAYS FROM EXTRACELLULAR MATRIX
}

\author{
Wen Shen *, Laura Struzyna**, D. Kacy Cullen**, and Mark G. Allen* \\ *Singh Center for Nanotechnology, University of Pennsylvania, USA \\ **School of Medicine, University of Pennsylvania, USA
}

\begin{abstract}
This paper reports a suite of microfabrication strategies that allow electronic structures to be fabricated on and within protein extracellular matrix (ECM)-based substrates. ECM-based implantable neural electrodes (NEs) are presented here as a case study. ECM proteins are processed to form flexible substrates to promote neural growth and function. A stiff delivery needle or needle array is integrated with the ECM-NEs to facilitate successful insertion and is subsequently removed. Preliminary animal validation experiments using larger ECM-NEs showed in vivo intracortical recording capabilities of the ECM-NEs from rat motor cortex.
\end{abstract}

\section{INTRODUCTION}

Advances in neural microelectrodes (NEs) have enabled interfacing with and extracting information from neural circuits [1] and have led to many significant advances such as neural recordings, deep brain stimulation, and brain-controlled prosthetic devices [2, 3]. Current state-of-the-art NEs in use, particularly in clinical settings, are typically fabricated from silicon or noble metals [4]. These devices face two major challenges which hinder their sustainable communication with neurons over long periods of time. First, materials composing these devices are typically foreign to their implanted environment, resulting in a sustained glial response that eventually leads to neurodegeneration and decreases the fidelity of the recorded neural signals [5]. Second, the large mechanical mismatch between the soft neural tissue and the rigid devices results in large stress and strains and causes local inflammation at the device-tissue interfaces, which impede the sustained functionality of these devices [6].

To resolve these challenges, biomolecule-based coatings including ECM molecules [7] and anti-inflammatory drugs [8] have been implemented on NE surfaces, and showed initial improvement in promoting neural cell growth and preventing glial scar formation at the tissue-device interfaces, ultimately enhancing neuronal attachment and achieving improved initial recordings in vivo. However, these results were unsustainable at longer time scales and, thus, could not support chronic neural interfacing [9, 10]. Research suggested that reduction in the mechanical modulus of the materials comprising the device is expected to provide a more tissue-complaint interface, thereby lowering the strains induced on the tissue. However, soft/flexible NEs face a materials challenge because they must also be sufficiently stiff to penetrate into the neural tissue while minimizing stresses and damages. The mechanical compatibility [11] and biocompatibility [12] of ECMbased materials suggest that, in contrast to a coating, fabricating almost the entire NE from ECM material may be fruitful. We have recently developed ECM-NEs that are primarily composed of collagen [13]. Due to the neuronal compatibility and mechanical flexibility of collagen, the collagen-based NEs produced increased neural compatibility and reduced inflammatory responses compared with inorganic approaches. However, the dimensions of those collagen-based NEs were $310 \mu \mathrm{m}$ in width, which is an order of magnitude larger than the cortical neuron diameters. The relative large sizes of those ECM-NEs could result in significant injury to the neural environment during device implantation. To further reduce this mechanical injury, it is desirable to reduce the sizes of ECM-NEs to approach neuronal feature sizes. However, as sizes reduce, these flexible NEs may reach a mechanical limit that they cannot be sufficiently stiff for implantation. In these cases, an implantation tool is needed to deliver these ECM-NEs into the neural tissue. In addition, to further improve biocompatibility, it is desirable to fabricate NEs using laminin, a brain ECM protein that can provide better compatibility with the neuronal environment [14].

Here we reported a set of fabrication processes that allow the fabrication of ECM-NEs with width of $100 \mu \mathrm{m}$ using both the collagen substrate and a collagen-laminin composite substrate, where collagen can provide mechanical flexibility and toughness and laminin can allow improved neuronal compatibility. Moreover, these processes are integrated with a stainless steel delivery vehicle on the ECM-NEs to facilitate implantation of these smaller devices. Further, fabrication of ECM-NE arrays in both $2 \mathrm{D}$ and 3D conformations, desirable for high-spatial resolution neuronal interrogation over a larger volume of tissue, is enabled.

\section{DESIGN AND FABRICATION OF ECM-NE}

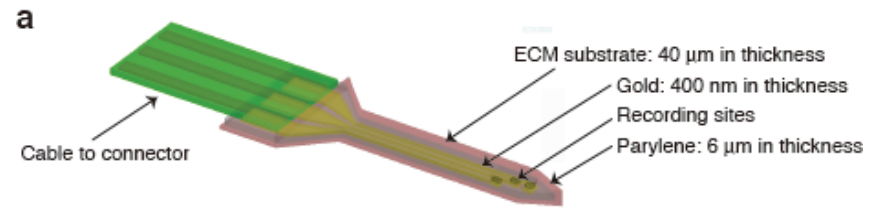

b

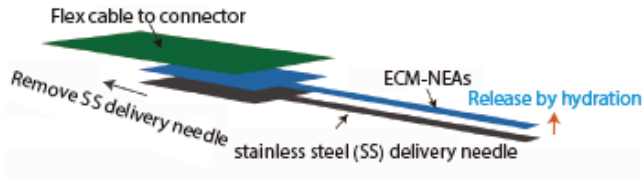

C

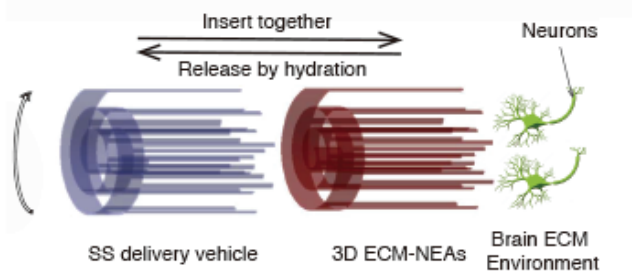

Figure 1: Schematic of (a) ECM-NEs, (b) ECM-NEs integrated with stainless steel delivery needle, and (c) rolling the $2 D$ array to $3 D$ arrays. The delivery needle is separated from the ECM-NEs and removed from the brain after implantation, leaving only ECMNEs implanted in the brain and interfacing with neurons.

The shape of each ECM-NE shank followed those of commercial Michigan electrodes that use a planar needle design. Each ECM-NE comprises an ultrathin ( $6 \mu \mathrm{m}$ in thickness) parylene microelectrode core embedded within a $40 \mu \mathrm{m}$ thick flexible ECM 
protein substrate as shown in Figure 1a. The ECM substrate provides a large amount of neuronal ECM matrix protein to promote neural growth and function and potentially minimize the inflammatory responses and biocompatibility concerns associated with the implanted devices. Previously fabricated collagen-NEs were $300 \mu \mathrm{m}$ in width, three times wider than the commercial Michigan arrays [4]. The relatively large size of those previous arrays could increase the mechanical injury to the tissue. In this work, we designed and developed ECM-NEs that are $100 \mu \mathrm{m}$ in width, comparable to the sizes of commercial Michigan arrays. To facilitate the implantation of these ECM-NEs, a thin $(25 \mu \mathrm{m})$ stainless steel needle layer was integrated with the ECM-NEs as shown in the schematic in Figure 1b. These stainless steel needles could be released from ECM-NEs and removed from the tissue after implantation of the ECM-NEs. To enable high spatial resolution neural interrogation over a larger volume of neural tissue, 3D needle arrays were achieved by rolling the linear 2D needle arrays around a hypodermic needle that defines the inner diameter of the 3D array (Figure 1c). In addition, these stainless steel needles also serve as shadow masks during the laser micromachining of ECM-NEs, eliminating errors generated during the alignment and positioning of the devices under the laser beam.

The fabrication processes of the ECM-NEs are illustrated in Figure 2. A thin ( $3 \mu \mathrm{m}$ in thickness) Parylene C (poly-monochloropara-xylylene) film was deposited on a silicon carrier wafer (Figure 2a) using a chemical vapor deposition process (SCS Labcoater, Special Coating Systems, Inc., Indianapolis, IN, USA). A $200 \mathrm{~nm}$ thick gold layer was deposited using electron beam evaporation and patterned by lift-off, yielding interconnecting lines and pads (Figure 2b). Another thin ( $3 \mu \mathrm{m}$ in thickness) layer of Parylene $\mathrm{C}$ was deposited and then patterned by reactive ion etching, leaving only the pads at the ends of the lines exposed (Figure 2c).

The preparation of collagen films that are ultimately used as substrates for further processing were described in [13]. The collagen-laminin composite film in this work was prepared as follows. Type I rat tail collagen in a $3 \mathrm{mg} \mathrm{mL}^{-1}$ solution (Corning ${ }^{\circledR}$, Corning, NY) was gently mixed with $10 \mathrm{X}$ phosphatebuffered saline (PBS), $0.1 \mathrm{M} \mathrm{NaOH}$ and $6 \mathrm{mg} \mathrm{mL}^{-1}$ laminin (Trevigen, Gaithersburg, MD) at a ratio of 13:2:1:1 by volume until small fragments started to form. The mixed solution was then cast into an acrylic mold, followed by polymerization at $37^{\circ} \mathrm{C}$ and $96 \%$ humidity for 24 hours, and then dried on glass slides in air at $37^{\circ} \mathrm{C}$ for $24 \mathrm{~h}$. This collagen-laminin composite film was rinsed with DI water multiple times until a fully transparent film was observed. After rinsing, the film was air dried, forming a uniform dried collagen-laminin composite film (thickness of $40 \mu \mathrm{m}$ ). This collagen-laminin composite film was subsequently removed from the acrylic mold and glass slides for subsequent use as substrates.

The first layer ECM (collagen, or collagen-laminin composite) substrate in sheet form was then applied under hydrated condition on top of the parylene core. After the ECM dehydrated, the parylene cores were embedded within the ECM substrate (Figure 2d). This ECM substrate with embedded parylene core was then peeled off from the silicon carrier wafer, flipped, and embedded with another layer of ECM hydrogel substrate. After the second layer of ECM substrate dehydrated, no obvious interface was found between the two ECM layers under SEM. This ECM substrate encapsulating the parylene core was then aligned, rehydrated, and dehydrated on top of a stainless steel layer that was previously patterned into $2 \mathrm{D}$ needle arrays using $532 \mathrm{~nm}$ green laser micromachining so that the collagen substrate is attached to the stainless steel (Figure 2e). This ECM substrate attached to the stainless steel substrate was then flipped, and patterned to form 2D arrays of ECM-NEs using a $193 \mathrm{~nm}$ excimer laser etching with the stainless steel serving as a contact shadow mask (Figure $2 \mathrm{f}$ ). The use of stainless steel contact mask eliminated the alignment of the ECM-NE patterns with the laser beam, thus avoided errors that would be generated during the otherwise needed alignment step.

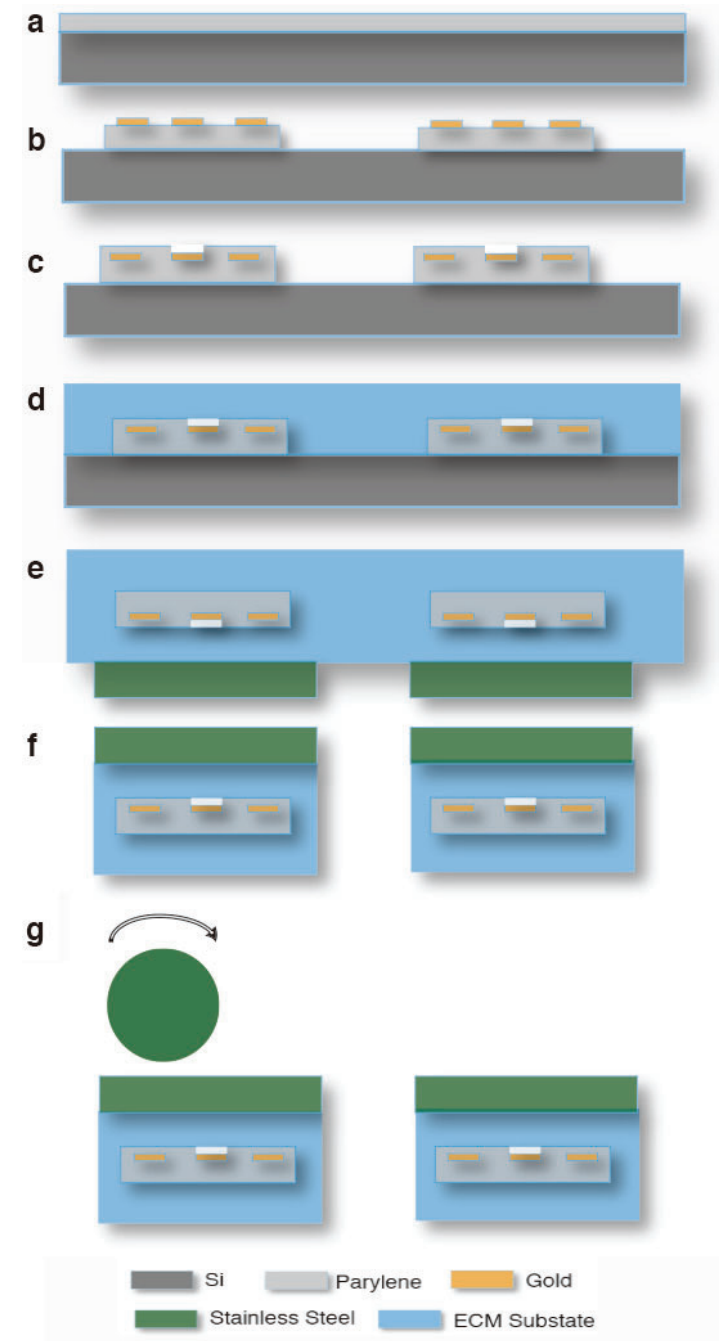

Figure 2: Fabrication processes of the ECM-NEs: (a) Parylene was deposit on Si carrier wafer, (b) gold traces were deposited and patterned using lift-off, (c) top layer parylene was deposit and patterned using reactive ion etching (RIE), (d) parylene core was embedded within ECM substrate, (e) ECM substrate with embedded parylene core was peeled off from the structure, flipped and encapsulate with a $2^{\text {nd }}$ layer ECM substrate, and then aligned on the pre-patterned stainless steel layer, (f) the ECM substrate attached to the stainless steel was flipped, and patterned to form a $2 D$ array of ECM-NEs using excimer laser etching with stainless steel serving as a contact shadow mask, and (g) 2D linear array was rolled around a hypodermic needle to form the $3 D$ array.

Dense 3D NE arrays are desired for high spatial resolution neural interrogation over a larger volume of tissue. To fabricate the 3D ECM-NE arrays (3D ECM-NEAs), we first fabricated long 2D linear arrays on a stainless steel insertion vehicle. A slit $(1 \mathrm{~mm}$ in 
width and $5 \mathrm{~mm}$ in length) was cut at the tip of the 26G hypodermic needle using a $532 \mathrm{~nm}$ green laser for use as a clip to hold the 2D linear array. This linear array was then rolled around the $26 \mathrm{G}$ hypodermic needle (Figure $2 \mathrm{~g}$ ) to form a $3 \mathrm{D}$ array with cylindrical shape.
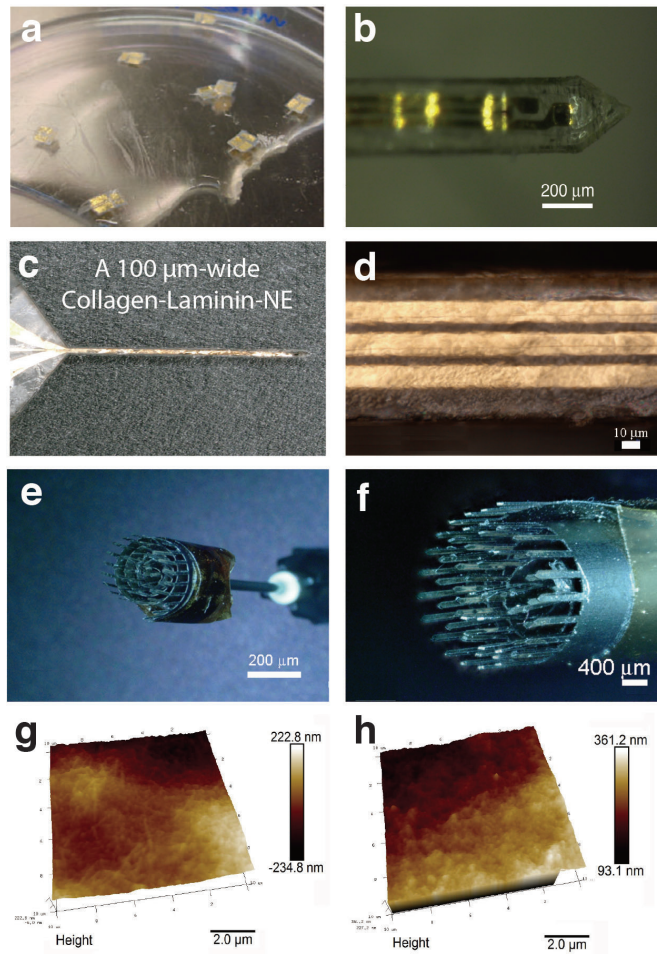

Figure 3: Fabricated ECM-NES and stainless steel delivery vehicles: (a) collagen-NE (shank width: $310 \mu \mathrm{m}$, shank length: 2 mm) floating in DI water, (b) tip of a collagen-NE (shank width: $310 \mu \mathrm{m}$, previous work), (c)-(d) collagen-laminin-NE (shank width: $100 \mu \mathrm{m}$, shank length: $5 \mathrm{~mm}$ ), (e) 3D array rolled around a $26 G$ hypodermic needle, (f) close-up view of the $3 D$ array, $(g)-(h)$ AFM height images of $(g)$ collagen substrate, and $(h)$ collagenlaminin composite substrate.

Figure 3 shows a representative picture of the fabricated devices. After being hydrated in DI water, the ECM-NEs kept their as-fabricated shape as shown in Figure 3a. No rapid degradation or change in shape was observed when the devices were immersed in DI water for 24 hours. The tip of a $310 \mu \mathrm{m}$ wide collagen-NE fabricated using previous method is shown in Figure 3b. Compared with the previous method, the method described herein allows fabrication of smaller ECM-NEs partly due to the reduced alignment error during the excimer etching of ECM substrate. Figure 3(c) and 3(d) show pictures of a fabricated $100 \mu \mathrm{m}$ wide and $5 \mathrm{~mm}$ long collagen-laminin NE using the new approach.

Laminin has long been recognized and used as a standard coating material to promote cortical neural regeneration, growth, and function [14]. However, laminin thin films are too fragile to sustain mechanical handling during microfabrication. To overcome this challenge, we have fabricated collagen I-laminin composite films. These collagen-laminin composite films exhibit increased toughness compared to laminin alone, and we found a collagen Ilaminin (13 wt\%) composite film is able to sustain the fabrication process described here without micro-cracks or damage manifesting in the ECM-NEs. This is due to the long fibrils and 3D structure of collagen I providing toughness to the composite films [11]. These collagen fibers normally have diameters of tens of nanometers [15]. Atomic force microscopy of the collagen substrate and collagen-laminin composite substrate show structure that is consistent with fibrous structures (Figure $3 \mathrm{~g}-\mathrm{h}$ ).

A dense 3D needle array is demonstrated in Figure 3(e) and $3(\mathrm{f})$. The inner diameter of the $3 \mathrm{D}$ array is defined by the diameter of the hypodermic needle tip, which is approximately $450 \mu \mathrm{m}$. The, spacing between two adjacent rolls is defined during the rolling process. Optionally, a sacrificial spacer material comprising a layer of PVA or dextran coated at the base of the linear array can assist in adjacent roll spacing.

\section{INSERTION OF ECM-NE}
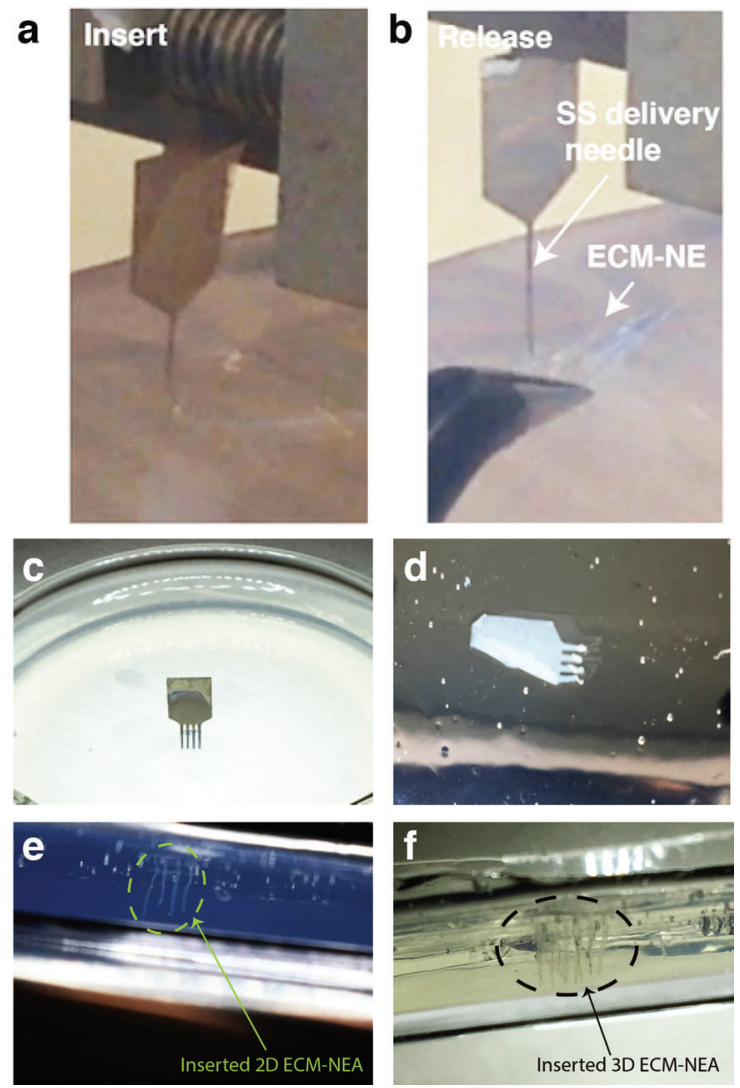

Figure 4: Insertion of ECM-NEs into a brain phantom material (1\% agarose gel). (a) insertion of a single shank ECM-NEs and (b) release and removal of the SS delivery needle after insertion, leaving only the single shank ECM-NE inserted; (c) insertion of a four-shank 2D ECM-NE array, and (d)-(e) after removing of the $2 D$ SS delivery needle, leaving only the four shanks ECM-NE inserted; (f) inserted $3 D$ ECM-NE arrays after removal of the $3 D$ SS delivery array.

The ECM-NEs were integrated with thin (thickness of $25 \mu \mathrm{m}$ ) microfabricated stainless steel (SS) needles to allow successful implantation into a brain mechanical model (Figure $4 \mathrm{a}$ and $4 \mathrm{c}$ ). Within one minute after implantation, the delivery needle was released from the ECM-NEs by hydration and was subsequently removed, leaving the flexible ECM-NEs at the implantation sites. Figure 4 (b) shows an inserted single shank ECM-NE, Figures 4 (d) and (e) show a top view and side view of a 4-shank 2D ECM$\mathrm{NE}$ array inserted in the brain phantom after release and removal 
of the 2D SS delivery array, and Figure 4 (f) shows the side view of a 18-shank 3D ECM-NE array inserted in the brain phantom after release and removal of the 3D-SS delivery array. These results demonstrated that the insertion method by fabricating and inserting the ECM-NEAs on SS delivery array together and subsequently removing the SS array upon insertion and hydration is a convenient and reliable method for insertion of ECM-NEA.

\section{ELECTRICAL FUNCTIONALITY OF ECM-NE}

We have previously shown that ECM-NEs are electrically functional and can record electrophysiological signals from rat barrel cortex upon whisker stimulation using larger ECM-NEs without insertion devices over a 54-day period (Figure 5a-c) [13]. These larger ECM-NEs supported square electrode sites $80 \mu \mathrm{m}$ on a side. When the sizes of the ECM-NEs are reduced, the electrode sizes must also be reduced. Here, we show that the ECM-NEs with diameter of $20 \mu \mathrm{m}$ show an impedance of approximately $500 \mathrm{k} \Omega$ at $1 \mathrm{kHz}$. This impedance agrees with the predicted model, and is within the allowable impedance range for NEs to record intracortical neuronal signals. Electroplating the NEs with nanoporous Pt could be considered to lower the device impedance for better signal to noise ratio [13].
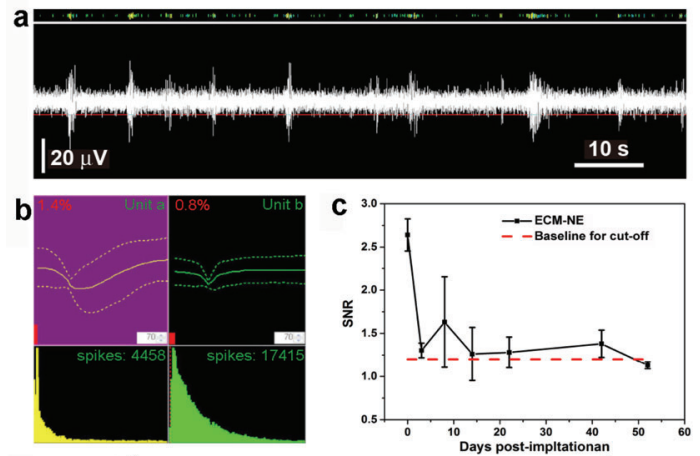

d

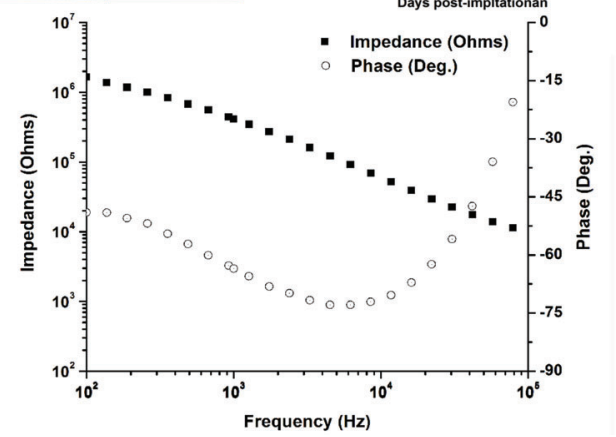

Figure 5: Electrical functionality of ECM-NEs. (a)-(c) In vivo electrophysiological neural signal recording data from previously fabricated ECM-NEs [4]. (a) Representative $90 \mathrm{~s}$ of high-speed recording showing neural spikes upon whisker stimulation, (b) mean waveform and ISI histogram, and (c) mean SNR of the largest signal over a 54-day time period. (d), EIS of ECM-NES with smaller (20 $\mu \mathrm{m}$ in diameter) recording sites.

Enabling microfabrication with ECM proteins is expected to lead to implantable microelectronics for optimum neuronal compatibility. Our current focus is on enabling microfabrication technologies to produce microelectronics with a broader range of ECM materials to enable optimal intracellular signaling and cell recruitment, tuning the mechanical properties of these devices to closely match those of the host tissue to minimize undesired stress and strains at the device-tissue interface, and advancing MEMS technologies to minimize device feature sizes to minimize the insertion trauma. The development of ECM-NEs is expected to resolve both materials and mechanical challenges associated with implantable NEs and support sustainable neural interfaces.

\section{REFERENCES}

[1] M. Velliste, S. Perel, M. C. Spalding, A. S. Whitford, and A. B. Schwartz, "Cortical control of a prosthetic arm for selffeeding," Nature, vol. 453, pp. 1098-1101, 2008.

[2] J. P. Donoghue, "Connecting cortex to machines: recent advances in brain interfaces," Nature Neuroscience, vol. 5, pp. 1085-1088, 2002.

[3] M. A. Nicolelis, "Brain-machine interfaces to restore motor function and probe neural circuits," Nature Reviews Neuroscience, vol. 4, pp. 417-422, 2003.

[4] J. C. Williams, R. L. Rennaker, and D. R. Kipke, "Long-term neural recording characteristics of wire microelectrode arrays implanted in cerebral cortex," Brain Research Protocols, vol. 4, pp. 303-313, 1999.

[5] R. V. Bellamkonda, S. B. Pai, and P. Renaud, "Materials for neural interfaces," MRS Bulletin, vol. 37, pp. 557-561, 2012.

[6] A. Sridharan, J. K. Nguyen, J. R. Capadona, and J. Muthuswamy, "Compliant intracortical implants reduce strains and strain rates in brain tissue in vivo," Journal of neural engineering, vol. 12, p. 036002, 2015.

[7] W. He, G. C. McConnell, and R. V. Bellamkonda, "Nanoscale laminin coating modulates cortical scarring response around implanted silicon microelectrode arrays," Journal of Neural Engineering, vol. 3, p. 316, 2006.

[8] W. He, G. C. McConnell, T. M. Schneider, and R. V. Bellamkonda, "A Novel Anti-inflammatory Surface for Neural Electrodes," Advanced Materials, vol. 19, pp. 35293533, 2007.

[9] X. Cui, J. Wiler, M. Dzaman, R. A. Altschuler, and D. C. Martin, "In vivo studies of polypyrrole/peptide coated neural probes," Biomaterials, vol. 24, pp. 777-787, 2// 2003.

[10] S. De Faveri, E. Maggiolini, E. Miele, F. De Angelis, F. Cesca, F. Benfenati, et al., "Bio-inspired hybrid microelectrodes: a hybrid solution to improve long-term performance of chronic intracortical implants," Frontiers in Neuroengineering, vol. 7, p. 7, 04/10

[11] M. P. Wenger, L. Bozec, M. A. Horton, and P. Mesquida, "Mechanical properties of collagen fibrils," Biophysical journal, vol. 93, pp. 1255-1263, 2007.

[12] Y. C. Huang and Y. Y. Huang, "Biomaterials and strategies for nerve regeneration," Artificial Organs, vol. 30, pp. 514$522,2006$.

[13] W. Shen, L. Karumbaiah, X. Liu, T. Saxena, S. Chen, R. Patkar, et al., "Extracellular matrix-based intracortical microelectrodes: Toward a microfabricated neural interface based on natural materials," Microsystems \& Nanoengineering, vol. 1, p. 15010, 06/29/online 2015.

[14] J. R. Sanes, "Extracellular matrix molecules that influence neural development," Annual review of neuroscience, vol. 12, pp. 491-516, 1989.

[15] D. E. Birk, J. M. Fitch, J. P. Babiarz, K. J. Doane, and T. F. Linsenmayer, "Collagen fibrillogenesis in vitro: interaction of types I and V collagen regulates fibril diameter," Journal of Cell Science, vol. 95, pp. 649-657, 1990.

\section{CONTACT}

*M. Allen, mallen@seas.upenn.edu 